\title{
DPP-4 inhibitors in the prevention/treatment of multi-organ injury caused by COVID-19 - a therapeutic approach of choice in type 2 diabetic patients?
}

\author{
Andrija Šmelcerović ${ }^{1}$, Gordana Kocić ${ }^{1}$, Mihajlo Gajić ${ }^{2}$, Katarina Tomović ${ }^{1}$, Vukica \\ -Dor ${ }^{-}$dević ${ }^{1}$, Dobrila Stanković -Dor ${ }^{-}$dević ${ }^{1}$, and Marko Anderluh ${ }^{3}$ \\ ${ }^{1}$ University of Nis Faculty of Medicine \\ ${ }^{2}$ Medicinal Faculty, University of Nis \\ ${ }^{3}$ Faculty of Pharmacy University of Ljubljana
}

May 6, 2020

\begin{abstract}
Since the outbreak of SARS-CoV-2 virus more than 2800000 cases have been reported worldwide. Patients suffering from diabetes and other comorbidities are particularly susceptible to severe forms of the COVID-19, which might result in chronic complications following recovery. Dipeptidyl peptidase-4 inhibitors exert beneficial effects in the prevention/treatment of pulmonary fibrosis, heart, and kidney injury, and since they may be a long-term consequence caused by COVID-19, it is reasonable to expect that DPP-4 inhibitors might be beneficial in alleviating long-term consequences of COVID-19. With that in mind, we would like to voice our concerns over chronic implications following recovery from COVID-19 especially in diabetic, but also in non-diabetic patients, and to indicate that some preventive measures could be undertaken by application of DPP-4 inhibitors.
\end{abstract}

\begin{abstract}
Since the outbreak of SARS-CoV-2 virus more than 2800000 cases have been reported worldwide. Patients suffering from diabetes and other comorbidities are particularly susceptible to severe forms of the COVID19, which might result in chronic complications following recovery. Dipeptidyl peptidase-4 inhibitors exert beneficial effects in the prevention/treatment of pulmonary fibrosis, heart, and kidney injury, and since they may be a long-term consequence caused by COVID-19, it is reasonable to expect that DPP-4 inhibitors might be beneficial in alleviating long-term consequences of COVID-19. With that in mind, we would like to voice our concerns over chronic implications following recovery from COVID-19 especially in diabetic, but also in non-diabetic patients, and to indicate that some preventive measures could be undertaken by application of DPP-4 inhibitors.
\end{abstract}

Keywords: COVID-19, diabetes, DPP-4 inhibitors

The end of SARS-CoV-2 pandemic is still behind the horizon, as the total confirmed cases number rapidly rises. As of April 27, 2020, more than 2800000 cases of SARS-CoV-2 infections have been reported out of which more than 198000 ended lethally (World Health Organization, 2020). The presence of chronic comorbidities may aggravate clinical findings and induce fatal outcome, as evidenced by the study on 44 672 confirmed cases, out of which 1023 had fatal outcome. Case fatality rates reported for patients suffering from chronic comorbidities including cardiovascular disease, diabetes, chronic respiratory disease, and hypertension $(10.5 \%, 7.3 \%, 6.3 \%$ and $6.0 \%$, respectively) were much higher compared to case fatality a rate 
of $0.9 \%$ for patients without comorbid conditions (The Novel Coronavirus Pneumonia Emergency Response Epidemiology Team, 2020). With the main focus of public health authorities on transmission prevention, development of vaccines and treatments, long-term consequences on the health of recovering and recovered patients remain subordinate. Radiological findings of COVID-19 patients indicate to pneumonia associated with rapidly developed pulmonary fibrotic changes, even in asymptomatic patients (Shi et al., 2020). Based on similarities between ongoing and previous coronavirus infections, excessive lung damage accompanied by fibrosis might result in functional disabilities decreasing quality of life among survivors (Ngai et al., 2010; Batawi et al., 2019). The evolution of pneumonia to pulmonary fibrosis may vary, but the interval between the onset of symptoms and the development of pulmonary fibrosis may be relatively short, sometimes even just a few days (Shi et al., 2020), which indicate an urgent therapeutic approach to preserve pulmonary function. Besides, multi-organ injury affecting liver, kidneys, and heart among others, that may eventually result in organ failure, is quite common among COVID-19 patients in intensive care units (Wang et al., 2020). SARS-CoV-2 either induces new cardiac pathologies, such as myocarditis, and/or leads to the exacerbation of existing ones (Madjid et al., 2020), implying potential long-term cardiovascular effects of COVID-19. This might be important especially for diabetic patients who already suffer from reduced lungs, hearth, and kidney function, making them particularly susceptible to cumulative organ injury during infection with SARS-CoV-2. With that in mind, we would like to voice our concerns over chronic implications following recovery from COVID-19 especially in diabetic, but also in non-diabetic patients, and to indicate that some preventive measures could be undertaken by application of DPP-4 inhibitors.

Amplified and aberrant immune response to SARS-CoV-2 infection causes pulmonary injury triggering defensive pro-fibrotic mechanisms, as a result of simultaneous tissue remodeling by activated myofibroblasts and migrating fibroblasts, which possess on their surface dipeptidyl peptidase-4 (DPP-4)/CD26. In fact, inflammation is tightly interconnected with fibroblast activation, migration, and proliferation. However, although host immune response has crucial role in COVID-19 pathology, opinions on the use of anti-inflammatory therapies, especially in critically ill patients, are conflicting (Ritchie and Singanayagam, 2020).

DPP-4 inhibitors are able to suppress inflammatory signalling and proliferation of vascular smooth muscle cells (Xu et al., 2018), which are important players in the reversible phase of pulmonary vascular remodelling. We have indicated to the possibility that DPP-4 inhibitors might prevent fibrosis and delay or suppress the entry to the irreversible phase of vascular remodeling in pulmonary hypertension by reducing the activity of pro-fibrotic mediators, proliferation, and migration of fibroblasts (Anderluh et al., 2019). These pleiotropic anti-inflammatory effects of DPP-4 inhibitors are incomparable to standard anti-inflammatory therapies (i.e. corticosteroids) and might be useful in the therapy of COVID-19 due to their other favorable effects, especially after the clinical findings suggesting the lack of benefit from corticosteroids, and even indicating to deleterious effects accompanied with late complications (Russel et al., 2020). Figure 1 depicts the proposed mechanism of beneficial effects of DPP-4 inhibition on SARS-CoV-2 damaged lungs and other organs.

In addition, we have pointed to many beneficial pleiotropic effects of DPP-4 inhibitors with protective role in renal and cardiovascular disorders as specific entities, or type 2 diabetes mellitus associated comorbidities including myocardial regeneration. DPP-4 inhibitors exert these effects by reducing degradation of stromal cell-derived factor-1, which enhances homing of endothelial progenitor cells and ameliorates functional myocardial reparation outcome (Anderluh et al., 2016; Tomovic et al., 2019).

Recent reports clearly indicate that both hypertension and diabetes represent special risk factor as comorbidities with COVID-19 (The Novel Coronavirus Pneumonia Emergency Response Epidemiology Team, 2020). Accordingly, Fang, Karakiulakis et al. (2020) continued to suggest that patients with hypertension, who are prescribed either ACE inhibitors or angiotensin receptor antagonists known to increase ACE2 expression, might be at greater risk for severe COVID-19 infection and, therefore, should be monitored for ACE2-modulating medications. In contrast with ACE2-modulating medications, we have offered hypothesis that DPP-4 inhibitors act in prevention/treatment of pulmonary fibrosis, heart, and kidney injury, and since they may be a long-term consequence caused by COVID-19, it is reasonable to expect that DPP-4 inhibitors might be beneficial in alleviating long-term consequences of COVID-19. Since DPP-4 inhibitors are one of 
the mainstream medications for the treatment of diabetes mellitus type 2, it would be rather undemanding to monitor the effect of DPP-4 inhibitors on long-term consequences on the health of COVID-19 recovering or recovered patients. We urge the scientific community to do exactly this.

In conclusion, the main purpose of this hypothesis is to raise the awareness that the end of SARS-CoV-2 pandemic will not necessarily be the end of our fight with COVID-19, since the long-term consequences of excessive lung, heart, and kidney injury in continuously increasing number of patients are yet to be seen. Favorable effects of DPP-4 inhibition in the protection and tissue regeneration in the lungs, heart, and kidneys might prove valuable in the prevention of long-term COVID-19 repercussions. Administration of DPP-4 inhibitors to COVID-19 patients with ongoing type 2 diabetes mellitus could be advisable, as they are capable to benefit from effects on glycemic control, but also from protective anti-inflammatory, antiproliferative and anti-fibrotic effects in the lungs, hearth, and kidneys. At the end of twentieth century the concept of "one molecule-one target-one disease" shifted towards multitarget drugs able to control complex diseases (Alcaro et al., 2019). DPP-4 inhibitors are capable of simultaneously treating both diabetes and its multiple complications. The mentioned effects of DPP-4 inhibitors are expected to be beneficial for some or even all SARS-CoV-2 infected patients and we propose them as potential adjunctive treatment option aimed to alleviate COVID-19 long-term complications, especially knowing that millions of people will likely be affected by COVID-19.

\section{References}

Alcaro, S., Bolognesi, M.L., García-Sosa, A.T., \& Rapposelli, S. (2019). Multi-target-directed ligands (MTDL) as challenging research tools in drug discovery: From design to pharmacological evaluation. Frontiers in chemistry, 7, 71 .

Anderluh, M., Kocic, G., Tomovic, K., Kocic, H., \& Smelcerovic, A. (2019). DPP-4 inhibition: A novel therapeutic approach to the treatment of pulmonary hypertension?. Pharmacology $\&$ therapeutics, 201, 1-7.

Anderluh, M., Kocic, G., Tomovic, K., Kocic, R., Deljanin-Ilic, M., \& Smelcerovic, A. (2016). Cross-talk between the dipeptidyl peptidase- 4 and stromal cell-derived factor-1 in stem cell homing and myocardial repair: potential impact of dipeptidyl peptidase-4 inhibitors. Pharmacology $\&$ therapeutics , 167, 100-107.

Batawi, S., Tarazan, N., Al-Raddadi, R., Al Qasim, E., Sindi, A., Johni, S. A., et al. (2019). Quality of life reported by survivors after hospitalization for Middle East respiratory syndrome (MERS). Health and quality of life outcomes , 17 (1), 101.

Fang, L., Karakiulakis, G., \& Roth, M. (2020). Are patients with hypertension and diabetes mellitus at increased risk for COVID-19 infection?. The Lancet Respiratory Medicine . DOI:https://doi.org/10.1016/S2213-2600(20)30116-8

Madjid, M., Safavi-Naeini, P., Solomon, S.D., \& Vardeny, O. (2020). Potential effects of coronaviruses on the cardiovascular system: a review. JAMA cardiology . doi:10.1001/jamacardio.2020.1286

Ngai, J.C., Ko, F.W., Ng, S.S., To, K.W., Tong, M., \& Hui, D. S. (2010). The long-term impact of severe acute respiratory syndrome on pulmonary function, exercise capacity and health status. Respirology , 15(3), 543-550.

Novel Coronavirus Pneumonia Emergency Response Epidemiology Team. (2020). The Epidemiological Characteristics of an Outbreak of 2019 Novel Coronavirus Diseases (COVID-19) in China. China CDC Weekly, 2(8): 113-122.

Ritchie, A.I., \& Singanayagam, A. (2020). Immunosuppression for hyperinflammation in COVID-19: a double-edged sword?. The Lancet . DOI:https://doi.org/10.1016/S0140-6736(20)30691-7

Russel, C.D., Millar, J.E., \& Baillie, J.K. (2020). Clinical evidence does not support corticosteroid treatment for 2019-nCoV lung injury. The Lancet . DOI:https://doi.org/10.1016/S0140-6736(20)30317-2 
Shi, H., Han, X., Jiang, N., Cao, Y., Alwalid, O., Gu, J., et al. (2020). Radiological findings from 81 patients with COVID-19 pneumonia in Wuhan, China: a descriptive study. The Lancet Infectous Diseases . DOI:https://doi.org/10.1016/S1473-3099(20)30086-4

Tomovic, K., Lazarevic, J., Kocic, G., Deljanin-Ilic, M., Anderluh, M., \& Smelcerovic, A. (2019). Mechanisms and pathways of anti-inflammatory activity of DPP-4 inhibitors in cardiovascular and renal protection. Medicinal research reviews, 39(1), 404-422.

Wang, D., Hu, B., Hu, C., Zhu, F., Liu, X., Zhang, J., et al. (2020). Clinical characteristics of 138 hospitalized patients with 2019 novel coronavirus-infected pneumonia in Wuhan, China. Jama, 323(11), 1061-1069.

World Health Organization. (2020). Coronavirus disease 2019 (COVID-19) Situation report - 98. Retrieved from: https://www.who.int/docs/default-source/coronaviruse/situation-reports/20200427-sitrep-98covid-19.pdf?sfvrsn=90323472_4

Xu, J., Wang, J., He, M., Han, H., Xie, W., Wang, H., et al. (2018). Dipeptidyl peptidase IV (DPP4) inhibition alleviates pulmonary arterial remodeling in experimental pulmonary hypertension. Laboratory Investigation, 98(10), 1333-1346.

\section{Figure legend}

Figure 1. Simplified mechanism of possible beneficial effects of DPP-4 inhibition, through its antiinflammatory, anti-proliferative and anti-fibrotic actions, that counteracts detrimental COVID-19 repercussions.

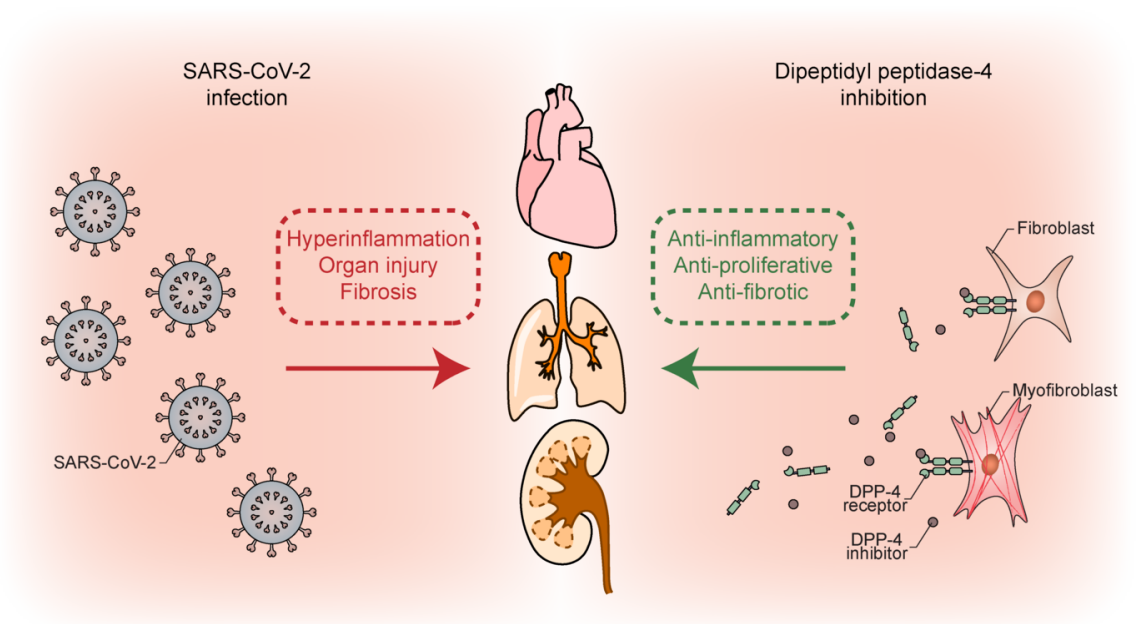

